\title{
28 Research Soure \\ Increased Expression of TSPEAR in Colorectal Cancer Predicts Poor Prognosis
}

\author{
Xintong Li \\ Jilin University \\ Yuandong Xie \\ Jilin University \\ Shuoyao Su \\ Jilin University \\ Zhe Liu \\ Jilin University \\ Jia Zhao \\ Jilin University \\ Dezhong Wen ( $\nabla$ wendz@jlu.edu.cn ) \\ Jilin University
}

\section{Research Article}

Keywords: TSPEAR, Colorectal cancer, Prognostic biomarker, Immune infiltration, Bioinformatic analysis

Posted Date: February 28th, 2022

DOl: https://doi.org/10.21203/rs.3.rs-1264778/v2

License: (c) (i) This work is licensed under a Creative Commons Attribution 4.0 International License. Read Full License 


\section{Abstract \\ Background}

Colorectal cancer (CRC) is, at present, the most frequently and most lethal gastrointestinal tract cancer, leading to significant mortality and a large global cancer burden. TSPEAR has been reported to associate with the development of human tooth and hair follicle morphogenesis. However, there is no study on TSPEAR in any cancers have been reported.

\section{Methods}

The information of TSPEAR gene expression in CRC was collected from TCGA database and GTEx. The RtqPCR and WB were used to compare the TSPEAR expression level between normal colon cells NCM-460 and human CRC cell lines, included SW-480, SW-620, HCT-116, Ht-29, and LoVo. Then, we performed the survival, gene function/pathway enrichment, and tumor immune infiltration analyses via using R packages and TIMER database.

\section{Results}

It was demonstrated that TSPEAR gene showed high expression in CRC tissue and cell lines and up-regulated expression correlating with a poor overall prognosis. Besides, the up-regulated TSPEAR was correlated with the M1 stage, CEA level, and residual tumor. Multivariate analysis further confirmed that TSPEAR remained an independent CRC patient-related risk factors, whereas gene function/pathway enrichment analysis indicated that it may can regulate Wnt, Hippo signaling pathway, as well as many cell metabolism activities. For immune infiltration, the expression level of TSPEAR was positively correlated with immune cells and showed a strong correlation with diverse immune marker sets, especially in COAD.

\section{Conclusions}

The results demonstrated that TSPEAR is possibly a clinically valuable predictive biomarkers for CRC patients, as well as associated with tumor infiltrating immune cells.

\section{Background}

Colorectal cancer (CRC), as the most frequently digestive tract malignant diseases, is the second leading cause of cancer related deaths globally, with an estimated 1.9 million new cases and over 935,000 deaths recorded alone in 2020(1). The primary treatment for CRC includes cancer-directed surgery, adjuvant or neoadjuvant therapies, systemic chemotherapy, radiation therapy, biologic therapy, and their combinations, which have significantly improved the therapeutic effects $(2,3)$. To date, the pathogenesis of CRC remains incompletely understood, and more effective treatment is also lacking. Therefore, the underlying molecular mechanisms of CRC development are urgently needed to be further investigated, and novel candidate 
biomarkers as well as new therapeutic targets for prognostic evaluation should be identified. Although some new biomarkers have been proposed as prognostic indicators, their clinical application is still limited due to tumor heterogeneity. Therefore, genetic and molecular profiling were used to identify new effective prognostic and predictive biomarkers for enhancing prognosis and individualized treatment.

Thrombospondin-type laminin G domain and EAR repeats (TSPEAR), locates on the chromosome 22 (22q22.3), whose function is poorly understood, especially in cancer field. Up to now, the study about how TSPEAR involves in occurrence and progression of cancer is lacking. However, previous studies have shown that TSPEAR was capable of regulating Notch signaling pathway, thus influence the development of human tooth and hair follicle morphogenesis, as well as dysmorphic features(4-6). The Notch signaling pathway is oncogenic, involved in regulating many cellular activities, including cell division, differentiation, apoptosis, as well as disease progression(7). The deregulation or mutation of Notch signaling could impact the tumorigenicity and the most hallmarks of cancer, especially, which have been highly noted in $\operatorname{CRC}(8,9)$. Herein, we evaluate the expression patterns, potential functions, and prognostic value of TSPEAR expression in $\mathrm{CRC}$, with reference to the bioinformatics analysis of gene expressions and relevant clinicopathologic parameters published online.

\section{Materials And Methods}

\section{Patient data sets}

The relevant gene expression data and clinical information (698 samples, HTSeq-FPKM) were retrieved from by the Colorectal Adenocarcinoma projects (COADREAD) in TCGA database (https://cancergenome.nih.gov), which is an available largescale publicly date portal of genome project, including 33 types of tumor sample information. The samples with insufficient survival information are not counted in this measurement. A total of 619 patients with CRC with the clinicopathological details and general features were acquired and further analyzed in this study.

\section{Cell culture}

The human normal colon cells NCM-460, human CRC cell lines SW-480, SW-620, HCT-116, Ht-29, LoVo, were purchased from the ATCC. Ht-29 and LoVo were cultured in RPMI-1640 medium (Gibco), other cell lines were cultured in DMEM (Gibco), all of which were supplemented with 10\% FBS (Gibco), and maintained at $37^{\circ} \mathrm{C}$ in a $5 \%$ CO2 humidified atmosphere.

\section{Real-time quantitative PCR of cell lines}

Extraction of total RNA from cell lines by SPARKeasy Improved Tissue/Cell RNA Kit (Sparkjade, \#AC0202). RNAs were reverse-transcribed into cDNA using SPARKscript $\triangle$ RT Plus Kit (Sparkjade, \#AG034). RT-qPCR was evaluated by the $2 \times$ SYBR Green qPCR Mix (with Rox) kit (Sparkjade, \#AH0104). The primer sequences were as follows: TSPEAR forward, 5- CGGTGGACATAATGGCCGAT-3, TSPEAR reverse, 5-

AGCACCTCGTTATCTTCTGGC -3, GAPDH was used as an internal control, and the expression level was analyzed using the $2-\triangle \triangle C T$ method. 


\section{Western blot}

Total protein from cells was extracted using RIPA lysis buffer (Beyotime, China) containing protease and phosphatase inhibitors. BCA Protein Assay Kit (Beyotime, China) was used for total protein quantification. The antibodies: TSPEAR antibody (1:1000 dilution, \#bs-9577R, Bioss), GAPDH antibody (1:5000 dilution, \#MB001, Bioworld Technology). The detailed steps were refered as $\mathrm{Li}$ et al(10). The results were analysed by Image $\mathrm{J}$ software.

\section{Functional enrichment analysis}

The function "cor.test" implemented in R with the Spearman method was utilized to filter the most positive or negative correlation coefficients with TSPEAR. Then, we perform GO and KEGG analysis by using "enrichGo" and "enrichKEGG" functions of the "clusterprofile.package". GO enrichment analysis was performed to predict the functional roles of TSPEAR gene, included BP, CO, MF. Similarly, KEGG pathways analysis was utilized to determine the pathways related toTSPEAR alternative functions and the frequently altered neighbor genes,

\section{Immune cell infiltration}

TIMER, a public available website (http:// cistrome.org/TIMER/), inclusion of 32 cancer types and 10,897 samples derived from the TCGA database(11). Herein, we mainly focused on the abundance of six types of infiltrating immune cells in CRC patients, included B cells, CD4+ T cells, CD8+ T cells, neutrophils, macrophages, and dendritic cells. Furthermore, we performed the correlation module analysis to explore relationships between TSPEAR expression and possibly gene markers of tumor-infiltrating immune cells, included CD $8+$ T cells, B cells, T-helper 1 (Th1) cells, -helper 2 (Th2) cells, T-helper 22 (Th22) cells, exhausted T cells, macrophages, TAMs, natural killer (NK) cells, and dendritic cells. Prior studies provide a reference for these gene markers $(12,13)$.

\section{Statistical analysis}

The R-3.6.3 was utilized to acquire and analyze statistic. Kaplan-Meier survival curves was used to estimate prognostic value of TSPEAR expression. The TSPEAR gene expression level in CRC patients was evaluated by box plots. The logstic regression was used to analyze accordingly correlation between clinicopathologic variables and TSPEAR expression in CRC. Besides, using a univariate Cox regression approach, with a multivariate Cox analysis, the influence of TSPEAR on survival and other clinicopathologic variables was estimated. A p-value less than 0.05 was considered statistically significant.

\section{Results}

\section{Baseline characteristics of patients}

As shown in Table 1, a total of 619 primary tumor cases with the corresponding clinical details and expression data were retained from TCGA data portal. Among the 619 patients, male patients were 343 (53.3\%), with female 289 (46.7\%). The median age was 68 years. In terms of pathologic stage, 105 patients 
were stage I (17.5\%), stage II in 227 (37.9\%), stage III in 179 (29.9\%), and stage IV in 88 (14.7\%). The neoplasm type included 454 patients with colon adenocarcinoma $(73.3 \%)$ and 165 rectum adenocarcinoma patients (74.0\%). Primary therapy outcomes of CRC included $12.8 \%$ in stable disease (SD) and progressive disease (PD), most patients were in partial remission (PR) and complete remission (CR)(87.3\%).

\section{High TSPEAR expression in CRC}

To assess the expression level of TSPEAR, the CRC samples from TCGA were compared normal tissues and GTEx databases. The results showed that TSPEAR gene expression level was significantly higher in CRC tissues $(p<0.001)$ than that in normal tissues (Figure 1A). The results were verified in CRC tissues and paired normal tissues $(p<0.001)$ (Figure 1B). Besides, TIMER database further verified the high expression of TSPEAR both in COAD and READ (Figure 1F). In term of cell lines, WB and Rt-qPCR both showed that TSPEAR was up-regulated in $\mathrm{CRC}$ cell lines (Figure 1C-1E).

\section{Correlation between TSPEAR expression and clinical features}

As shown in Figure 2, we have summarized the association between TSPEAR expression and various clinicopathologic variables of $619 \mathrm{CRC}$ patients. The results revealed the high TSPEAR expression was enriched in M1 stage $(p=0.036)$ (Figure $2 F), C E A$ level $<=5 \mathrm{ng} / \mathrm{ml}(p=0.008)$ (Figure $2 \mathrm{G})$, residual tumor $(R 1 \& R 2)(p=0.044)$ (Figure 2I). However, no significant difference was found in the TSPEAR expression patients in age $(p=0.504)$ (Figure $2 A)$, gender $(p=0.514)$ (Figure 2B), pathologic stage $(p=0.132)$ (Figure $2 C)$, T stage $(p=0.626)$ (Figure $2 D), N$ stage $(p=0.23)$ (Figure $2 E)$, primary therapy outcome $(p=0.143)$ (Figure 2H).

\section{High expression of TSPEAR is an independent risk factor for overall survival}

Based on the median expression of TSPEAR, 619 samples were divided into two groups of up-regulated TSPEAR expression and down-regulated TSPEAR expression. As shown in Figure 3A, Kaplan-Meier survival analysis revealed that CRC patients with up-regulated TSPEAR were associated with poorer overall prognosis than that with TSPEAR-low $(p=0.003)$. Besides, subgroup analysis based on different clinical variables indicated that high TSPEAR expression affected the overall survival significantly in CRC cases of age $>65$ years old $(p=0.011)$ (Figure 3B), male $(p=0.023)$ (Figure 3C), T3/T4 $(p=0.012)$ (Figure 3D), N1 $(p=0.028)$ (Figure 3E), M1 ( $p=0.048$ ) (Figure 3F), R0 ( $p=0.031$ ) (Figure $3 G)$ and CEA level $>5 \mathrm{ng} / \mathrm{ml}(p=0.013)$ (Figure $3 \mathrm{H})$.

In a univariate analysis, we found that up-regulated TSPEAR expression correlated significantly with poor overall survival (hazard ratio $[\mathrm{HR}]=1.731,95 \%$ confidence interval $[\mathrm{Cl}]=1.213-2.470, \mathrm{p}=0.003$ ) (Table 2). Multivariate analysis confirmed that, with an HR of $2.204(95 \% \mathrm{Cl}=1.141-4.256, \mathrm{p}=0.01)$, TSPEAR gene expression remained independently associated with overall survival in patients with CRC (Table 2) (Figure 4).

\section{Correlation and enrichment analyses}

TCGA data was used to perform an analysis of correlated genes between TSPEAR and other genes in CRC. Firstly, the top 50 genes most positively- or negatively-correlated with TSPEAR were shown in the heatmap 
(Figure 5E, 5F). For gene function enrichment analysis, the top 300 genes correlated most positively with TSPEAR were selected. GO analysis shows that TSPEAR is significantly associated with positive regulation of peptide hormone secretion, left/right axis specification, phospholipase A2 activity (consuming 1,2-dipalmitoylphosphatidylcholine), integral component of presynaptic membrane, etc. (Figure 5A-5C). In addition, KEGG pathway analysis indicated the enrichments in the Wnt signaling pathway, Hippo signaling pathway, Arachidonic acid metabolism, and alpha-Linolenic acid metabolism (Figure 5D).

\section{Correlation between immune infiltration and TSPEAR}

Previous studies have shown that tumor-infiltrating lymphocytes serve as an indicator of TNM stage and survival in $\mathrm{CRC}(14)$. Therefore, we assessed the relationship between TSPEAR expression and immune infiltration levels in colon adenocarcinoma (COAD) and rectal adenocarcinoma (READ) from TIMER. As shown in figure $6 \mathrm{~A}$, the results displayed that TSPEAR expression has negative correlation with infiltrating levels of $B$ cells $(r=-0.108, P=3.00 e-02)$, CD8+ T cells $(r=-0.201, P=4.34 e-05)$, neutrophils $(r=-0.123, P=$ 1.35e-02), and dendritic cells $(r=-0.117, P=1.88 \mathrm{e}-02)$, while positively with CD4+ T cells $(r=0.12, P=1.61 \mathrm{e}-$ $02)$ in COAD. However, in terms of READ, only neutrophils $(r=-0.176, P=3.94 e-02)$ is negative with the expression of TSPEAR. In addition, TSPEAR expression has no significant association with macrophages in COAD and no correlations with tumor purity, B cells, CD8 + T cells, CD4+ T cells, macrophages, neutrophils, and dendritic cells in READ. $P<0.05$ was considered as the difference is of significance.

Above results show that TSPEAR is involved in tumor immune regulation in CRC, especially in COAD. To further exploration, the TIMER databases were used to execute the association between TSPEAR and specific markers of immune cell both in COAD and READ. As shown in table 3, the results demonstrated that the TSPEAR expression level was correlated with most immune marker genes of immune cells in COAD, included CD8 + T cells, different functional T cells, natural killer cell, as well as dendritic cell (Figure 6B-6G). However, the TSPEAR expression level was only correlated with 4 immune marker sets in READ (Table 2). These findings further verified that TSPEAR is associated with immune infiltrating in COAD.

\section{Discussion}

TSPEAR is a gene never reported in cancer. It has been shown that TSPEAR is expressed in many tissues such as the liver, intestine, lung, kidney, and testis (15). However, the function of TSPEAR is still poorly understood. One study proposed that TSPEAR gene was capable of encoding a member of the EpilepsyAssociated Repeat protein family, which play an important role during the development of the auditory system(15). In addition, the wild-type TSPEAR was detected at the plasma membrane, which indicates that TSPEAR is an extracellular protein and the presence of a ubiquitous TSPEAR receptor at the cell surface. But how the TSPEAR regulating normal cell and tumor cell growth and differentiation is still unclear. Therefore, the function role of FCGBP in cancer development and treatment is urgent to be clarified.

Herein, we proposed that TSPEAR gene expression level was associated with prognosis in CRC. An upregulated TSPEAR expression can be reckoned an independent prognostic biomarker for poor prognosis. Furthermore, more analysis results demonstrated that TSPEAR expression was correlated with several clinicopathologic features, included M stage, CEA level, as well as residual tumor. Furthermore, according to 
GO analyses, we found that TSPEAR expression phenotype was associated with the hormone regulation, component of presynaptic membrane and phospholipase A2 activity, however, all of which were the first to be studies, the specific mechanism needed to be further elucidated. The KEGG results show that TSPEAR expression was associated with many cell metabolism activities, such as Arachidonic acid metabolism, alpha - Linolenic acid metabolism, and Linoleic acid metabolism, and many oncogenic signaling pathways, such as Wnt signaling pathway and Hippo signaling pathway. The Wnt is a core cancer signaling pathway, which are often aberrantly regulated in a large variety of cancers(16). Especially, an increasing studies have demonstrated that Wnt signaling pathway was very essential during the development of human $\mathrm{CRC}(17)$. Besides, previous study has reported that Hippo signaling pathway was involved in diverse physiological processes, spanning from cell growth, proliferation, metabolism and tissue homeostasis to tumor formation and progression(18). These findings indicate that TSPEAR may influence tumor development through regulating canonical Wnt and Hippo signaling pathway. However, More specific studies are is needed for confirmation.

Tumor-infiltrating lymphocytes and tumor microenvironment immune cells were the key factors in tumor development, such as tumorigenesis, angiogenesis, tumor cell growth, and tumor metastasis(19). Immunotherapeutic strategies were reckoned as a potential direction for predicting survival status and therapeutic efficacy for tumor patients(20). Anti-programmed death-1 (PD-1) and anti-programmed death ligand-1 (PD-L1) have showed significantly treatment potential in advanced CRC especially(21, 22). Herein, our analysis reveals that different types of immune-infiltrating cells and specific markers of immune cell are correlated with TSPEAR expression in CRC, mainly in COAD, the mechanism of which is still unclear. Firstly, most of the T cells gene markers, such as CD8A of CD8 + T cells, TBX21, STAT1 and IFNG of Th1, STAT6 and STAT5A of Th2, CCR10, AHR of Th22, were inversely correlated with TSPEAR expression in COAD. These results reveal a potential mechanism that TSPEAR may influence COAD progression by regulating $T$ cell functions. In addition, gene markers of NK cell markers, such as KIR2DL1, KIR2DL4, KIR3DL2, KIR3DL2, and CD7, showed moderate and strong correlations with TSPEAR expression, which indicate that TSPEAR is a significant factor that relate to immune infiltration of NK cells. Furthermore, we observed significant correlations between TSPEAR and gene markers of Dendritic cell monocytes, such as HLA-DRA and HLADPA1, which further assess the potential regulating role of TSPEAR in polarization of tumor-associated macrophages (TAM), indicating that TSPEAR may be involved in regulating immune infiltration role in COAD microenvironment. Based on above findings, what we can be quite certain of, however, is that TSPEAR is capable of influencing immune cell infiltration and can be reckoned as a potential cancer biomarker.

\section{Conclusion}

All these findings identified that TSPEAR expression was up-regulated in CRC tissues and cell lines. Moreover, TSPEAR can be seen as an independent promising prognostic biomarker in patients with COAD. In addition, TSPEAR is certainly involved in immune infiltration to regulate CRC development. Therefore, elucidating a more specific molecular mechanism is of necessity, which can provide novel insights and therapeutic strategies to improve the long-term CRC treatments.

\section{Abbreviations}


CRC

colorectal cancer

COAD

colon adenocarcinoma

READ

rectal adenocarcinoma Rt-qPCR:real-time quantitative PCR

WB

western blot

SD

stable disease

PD

progressive disease

PR

partial remission

CR

complete remission

OS

Overall survival

TAM

tumor-associated macrophages

\section{Declarations}

\section{Acknowledgements}

Not applicable.

\section{Authors' contributions}

DZW and JZ designed and revised the manuscript. XTL drafted the manuscript. XTL and YDX prepared the figures and tables, SYS and ZL participated in the drafting and editing of the manuscript. All authors read and approved the final manuscript.

\section{Funding}

Not applicable.

\section{Availability of data and materials}

The raw data was downloaded from TCGA database (https://cancergenome.nih.gov). All data are publicly accessible. All data generated or analyzed during this study are included in this published article. 
Ethical approval is not required because this analysis was performed on public database and does not involve any studies with human participants or animals which were performed by the authors. The original research ethics information can be accessed in TCGA database (https://cancergenome.nih.gov).

\section{Consent for publication}

Consent for publication was obtained from all authors.

\section{Competing interests}

The authors declare that they have no conflict of interest.

\section{References}

1. Sung H, Ferlay J, Siegel RL, Laversanne M, Soerjomataram I, Jemal A, et al. Global Cancer Statistics 2020: GLOBOCAN Estimates of Incidence and Mortality Worldwide for 36 Cancers in 185 Countries. CA Cancer J Clin. 2021,71(3):209-49.

2. Chen W, Sun K, Zheng R, Zeng H, Zhang S, Xia C, et al. Cancer incidence and mortality in China, 2014. Chin J Cancer Res. 2018,30(1):1-12.

3. LH B, D S. Diagnosis and Treatment of Metastatic Colorectal Cancer: A Review. JAMA. 2021,325(7):66985.

4. Song JS, Bae M, Kim JW. Novel TSPEAR mutations in non-syndromic oligodontia. Oral Dis. 2020,26(4):847-9.

5. Peled A, Sarig O, Samuelov L, Bertolini M, Ziv L, Weissglas-Volkov D, et al. Mutations in TSPEAR, Encoding a Regulator of Notch Signaling, Affect Tooth and Hair Follicle Morphogenesis. PLoS Genet. 2016,12(10):e1006369.

6. Bowles B, Ferrer A, Nishimura CJ, Pinto EVF, Rey T, Leheup B, et al. TSPEAR variants are primarily associated with ectodermal dysplasia and tooth agenesis but not hearing loss: A novel cohort study. Am J Med Genet A. 2021.

7. Previs RA, Coleman RL, Harris AL, Sood AK. Molecular pathways: translational and therapeutic implications of the Notch signaling pathway in cancer. Clin Cancer Res. 2015,21(5):955-61.

8. Tyagi A, Sharma AK, Damodaran C. A Review on Notch Signaling and Colorectal Cancer. Cells. 2020,9(6).

9. Vinson KE, George DC, Fender AW, Bertrand FE, Sigounas G. The Notch pathway in colorectal cancer. Int J Cancer. 2016,138(8):1835-42.

10. Li WX ZJ, Zhao G, Lyu CT, Lu WQ. Overexpression of DSCR1 prevents proliferation and predicts favorable prognosis in colorectal cancer patients. World J Surg Oncol 2021,19(1):100.

11. Taiwen Li, Jingyu Fan, Binbin Wang, Nicole Traugh, Qianming Chen, Jun S Liu, et al. TIMER: A Web Server for Comprehensive Analysis of Tumor-Infiltrating Immune Cells. Cancer Res. 2017,77(21):e108-e10.

12. Nathan $O$ Siemers, James L Holloway, Han Chang, Scott D Chasalow, Petra B Ross-MacDonald, Charles F Voliva, et al. Genome-wide association analysis identifies genetic correlates of immune infiltrates in solid tumors. PLoS One. 2017,12(7):e0179726. 
13. Patrick Danaher, Sarah Warren, Lucas Dennis, Leonard D'Amico, Andrew White, Mary L Disis, et al. Gene expression markers of Tumor Infiltrating Leukocytes. J Immunother Cancer. 2017,5(18).

14. Guo L, Wang C, Qiu X, Pu X, Chang P. Colorectal Cancer Immune Infiltrates: Significance in Patient Prognosis and Immunotherapeutic Efficacy. Front Immunol. 2020,11:1052.

15. Delmaghani S, Aghaie A, Michalski N, Bonnet C, Weil D, C P. Defect in the gene encoding the EAR/EPTP domain-containing protein TSPEAR causes DFNB98 profound deafness. Hum Mol Genet. 2012,21(17):3835-44.

16. Yann Duchartre, Yong-Mi Kim, Kahn M. The Wnt signaling pathway in cancer. Crit Rev Oncol Hematol. 2016,99:141-9.

17. Xiaofei Cheng, Xiangming Xu, Dong Chen, Feng Zhao, Wang W. Therapeutic potential of targeting the Wnt/ $\beta$-catenin signaling pathway in colorectal cancer. Biomed Pharmacother . 2019,110:473-81.

18. Noa Furth, Yael Aylon, Oren M. p53 shades of Hippo. Cell Death Differ. 2017,25(1):81-92.

19. Caro GD, Marchesi F, Laghi L, Grizzi F. Immune cells: plastic players along colorectal cancer progression. J Cell Mol Med. 2013,17(9):1088-95.

20. Greten FR, Grivennikov SI. Inflammation and Cancer: Triggers, Mechanisms, and Consequences. Immunity. 2019,51(1):27-41.

21. Overman MJ, McDermott R, Leach JL, Lonardi S, Lenz H-J, Morse MA, et al. Nivolumab in patients with metastatic DNA mismatch repair-deficient or microsatellite instability-high colorectal cancer (CheckMate 142): an open-label, multicentre, phase 2 study. The Lancet Oncology. 2017,18(9):1182-91.

22. Pan JH, Zhou H, Cooper L, Huang JL, Zhu SB, Zhao XX, et al. LAYN Is a Prognostic Biomarker and Correlated With Immune Infiltrates in Gastric and Colon Cancers. Front Immunol. 2019,10:6.

\section{Tables}


Table 1. Clinical characteristics of the colorectal adenocarcinoma patients.

\begin{tabular}{|c|c|c|c|}
\hline Clinical characteristics & & $\mathrm{N}$ & (\%) \\
\hline Age (years) & $<=65$ & 269 & $43.5 \%$ \\
\hline & $>65$ & 350 & $56.5 \%$ \\
\hline Gender & Female & 289 & $46.7 \%$ \\
\hline & Male & 330 & $53.3 \%$ \\
\hline Race, n (\%) & Asian & 12 & $3.3 \%$ \\
\hline & Non-Asian & 357 & $96.7 \%$ \\
\hline Pathologic stage & Stage I & 105 & $17.5 \%$ \\
\hline & Stage II & 227 & $37.9 \%$ \\
\hline & Stage III & 179 & $29.9 \%$ \\
\hline & Stage IV & 88 & $14.7 \%$ \\
\hline T stage & $\mathrm{T} 1 / \mathrm{T} 2$ & 125 & $20.2 \%$ \\
\hline & $\mathrm{T} 3 / \mathrm{T} 4$ & 492 & $79.8 \%$ \\
\hline N stage & No & 351 & $57 \%$ \\
\hline & N1 & 150 & $24.4 \%$ \\
\hline & $\mathrm{N} 2$ & 115 & $18.7 \%$ \\
\hline M stage, n (\%) & Mo & 459 & $84.1 \%$ \\
\hline & M1 & 87 & $15.9 \%$ \\
\hline Neoplasm type & Colon adenocarcinoma & 454 & $73.3 \%$ \\
\hline & Rectum adenocarcinoma & 165 & $26.7 \%$ \\
\hline Primary therapy outcome & $P D+S D$ & 38 & $12.8 \%$ \\
\hline & $P R+C R$ & 259 & $87.3 \%$ \\
\hline Residual tumor & Ro & & 450 (91.5\%) \\
\hline & $\mathrm{R} 1$ & & $6(1.2 \%)$ \\
\hline & R2 & & $36(7.3 \%)$ \\
\hline CEA level, n (\%) & $<=5$ & & $252(63.5 \%)$ \\
\hline & $>5$ & & 145 (36.5\%) \\
\hline History of colon polyps, $\mathrm{n}(\%)$ & No & & $364(68.4 \%)$ \\
\hline & Yes & & $168(31.6 \%)$ \\
\hline
\end{tabular}


Table 1. Clinical characteristics of the colorectal adenocarcinoma patients.

Clinical characteristics

Colon polyps present, $\mathrm{n}(\%)$

OS event, $\mathrm{n}(\%)$
$\mathrm{N}$

No

Yes

Alive

Dead
(\%)

$207(69.5 \%)$

$91(30.5 \%)$

$492(79.5 \%)$

$127(20.5 \%)$

SD: stable disease; PD: progressive disease; PR: partial remission; CR: complete remission; OS: overall survival; NRD: no residual disease; RD: residual disease.

Table 2. Univariate and multivariate Cox regression analyses of clinical characteristics associated with overall survival.

\begin{tabular}{|c|c|c|c|c|c|c|}
\hline \multirow[t]{2}{*}{ Clinical Characteristics } & \multicolumn{4}{|c|}{ Univariate analysis } & \multicolumn{2}{|c|}{ Multivariate analysis } \\
\hline & $\mathrm{HR}$ & $95 \% \mathrm{Cl}$ & $P$ value & $\mathrm{HR}$ & $95 \% \mathrm{Cl}$ & $P$ value \\
\hline Age & 1.939 & $1.320-2.849$ & $<0.001$ & 2.855 & $1.385-5.886$ & 0.004 \\
\hline Gender & 1.054 & $0.744-1.491$ & 0.769 & & & \\
\hline Race & 0.840 & $0.205-3.438$ & 0.809 & & & \\
\hline Pathologic stage & 2.988 & $2.042-4.372$ & $<0.001$ & 7.940 & $1.741-36.214$ & 0.007 \\
\hline T stage & 2.468 & $1.327-4.589$ & 0.004 & 2.984 & $0.680-13.093$ & 0.147 \\
\hline N stage & 2.627 & $1.831-3.769$ & $<0.001$ & 0.262 & $0.071-0.967$ & 0.044 \\
\hline M stage & 3.989 & 2.684-5.929 & $<0.001$ & 1.230 & $0.475-3.182$ & 0.670 \\
\hline Neoplasm type & 0.799 & $0.519-1.230$ & 0.308 & & & \\
\hline CEA level & 2.620 & $1.611-4.261$ & $<0.001$ & 1.706 & $0.846-3.439$ & 0.135 \\
\hline Residual tumor & 4.609 & $2.804-7.577$ & $<0.001$ & 2.114 & $0.925-4.831$ & 0.076 \\
\hline History of colon polyps & 0.789 & $0.496-1.257$ & 0.319 & & & \\
\hline Colon polyps present. & 1.250 & $0.743-2.103$ & 0.401 & & & \\
\hline TSPEAR & 1.731 & $1.213-2.470$ & 0.003 & 2.204 & $1.141-4.256$ & 0.019 \\
\hline
\end{tabular}


Table 3. Correlation analysis between TSPEAR and relate genes and markers of immune cells in TIMER.

\section{Description}

Gene

COAD

None

Cor P

CD $8+\mathrm{T}$ cell

B cell

Tfh

fh $\quad B C L 6$

-0.136 **

CD8B

CD19

CD20

CD38

BCL6

$-0.026$

$0.034 \quad 0.465$

ICOS

CXCR5

T-bet

STAT4

WSX1

STAT1

IFN-y

Th2

STA

Th22

Th22

T cell

exhaustion

LAGS

Macrophage

GATA3

CCR3

STAT6

STAT5A

$0.001 \quad 0.976$

$-0.043 \quad 0.362$

$-0.041 \quad 0.384$

0.578

$-0.129$

**

$-0.04$

0.398

-0.104 *

$-0.072$

Purity

Cor P

$-0.114$

*

0.048

0.331

0.063

0.202

$-0.046$

0.352

0.006

0.0912

$-0.007$

0.889

$-0.116$

*

$0.012 \quad 0.805$

\section{READ}

None

Cor P Cor P

$-0.083 \quad 0.0286$

$\begin{array}{ll}-0.139 & 0.0103\end{array}$

$\begin{array}{llll}0.044 & 0.577 & 0.016 & 0.855\end{array}$

$\begin{array}{llll}-0.024 & 0.764 & -0.098 & 0.252\end{array}$

-0.205 ** -0.186 *

$\begin{array}{llll}-0.034 & 0.668 & -0.08 & 0.347\end{array}$

$-0.026 \quad 0.578$

0.005

0.95

$-0.106$

0.176

$\begin{array}{ll}-0.04 & 0.607\end{array}$

-0.174 *

CCR10

$-0.072$

0.149

$-0.09$

0.149

$-0.06$

0.44

$\begin{array}{ll}-0.04 & 0.639\end{array}$

$0.059 \quad 0.207$

0.089

0.0707

$\begin{array}{ll}-0.112 & 0.151\end{array}$

$\begin{array}{lll}0.0745 & 0.042 \quad 0.595\end{array}$

$-0.12$

*

$-0.134$

$-0.196$

$-0.112$

*

$+$

0.15

$-0.008$

$-0.037 \quad 0.455$

$-0.081 \quad 0.299$

$\begin{array}{ll}-0.064 & 0.454\end{array}$

$-0.056 \quad 0.232$

$0.074 \quad 0.116$

0.09

*

0.0126 *

$-0.003 \quad 0.965$

0.226

$\star \star \star$

0.171

$\star \star \star$

0.092

0.241

$-0.043 \quad 0.583$

$-0.137$

0.107

0.194

AHR

$-0.288$

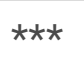

$-0.304$

$\star \star \star$

$-0.254$

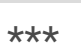

0.041

0.633

PD-1

0.01

0.844

$-0.038$

0.411

$-0.063$

3

0.42

$-0.241 \quad * \star \star$

GZMB

$0.234 * \star \star$

$\begin{array}{clllll}0.251 & 0.432 & 0.427 & * * * & 0.42 & * * * \\ -0.083 & 0.0932 & -0.125 & 0.11 & -0.162 & 0.0564 \\ -0.092 & 0.0632 & -0.088 & 0.259 & -0.121 & 0.155\end{array}$

TIM-3

-0.112 *

-0.101 *

CD11b

TAM

CCL2

$0.019 \quad 0.679$

0.052

$0.297 \quad 0.01$

$-0.003 \quad 0.944$

$\begin{array}{lllllll}0.036 & 0.463 & -0.069 & 0.378 & -0.072 & 0.4\end{array}$

$-0.048 \quad 0.305$

$-0.033$

$\begin{array}{lllll}0.508 & -0.005 & 0.947 & 0.004 & 0.964\end{array}$ 


\begin{tabular}{|c|c|c|c|c|c|c|c|c|c|}
\hline & CD86 & -0.097 & * & -0.08 & 0.109 & -0.133 & 0.0877 & -0.148 & 0.0811 \\
\hline \multirow{5}{*}{$\begin{array}{l}\text { Natural killer } \\
\text { cell }\end{array}$} & KIR2DL1 & -0.121 & ** & -0.123 & ** & 0.056 & 0.477 & 0.089 & 0.298 \\
\hline & KIR2DL4 & -0.242 & $\star \star \star \star ~$ & -0.24 & $\star \star \star \star ~$ & -0.052 & 0.503 & -0.093 & 0.274 \\
\hline & KIR3DL2 & -0.126 & $\star \star$ & -0.069 & 0.163 & -0.146 & 0.0609 & -0.14 & 0.101 \\
\hline & CD7 & -0.118 & * & -0.076 & 0.125 & -0.006 & 0.941 & -0.043 & 0.616 \\
\hline & CD141 & 0.078 & 0.0974 & 0.124 & * & 0.04 & 0.608 & 0.107 & 0.211 \\
\hline \multirow{2}{*}{$\begin{array}{l}\text { Dendritic } \\
\text { cell }\end{array}$} & HLA-DRA & -0.186 & $\star \star \star ~$ & -0.168 & $\star \star \star$ & -0.136 & 0.0816 & -0.147 & 0.0842 \\
\hline & $\begin{array}{l}\text { HLA- } \\
\text { DPA1 }\end{array}$ & -0.111 & * & -0.078 & * & -0.103 & 0.185 & -0.085 & 0.320 \\
\hline \multicolumn{10}{|c|}{$\begin{array}{l}\text { CODA, colon adenocarcinoma; READ, rectal adenocarcinoma; TAM, tumor-associated macrophage; Th, T } \\
\text { helper cell; T Th, Follicular helper T cell; Treg, regulatory T cell; Cor, R value of Spearman's correlation; None, } \\
\text { correlation without adjustment. Purity, correlation adjusted by purity. }\end{array}$} \\
\hline \multicolumn{10}{|c|}{ 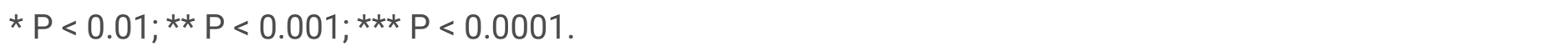 } \\
\hline
\end{tabular}

\section{Figures}


A

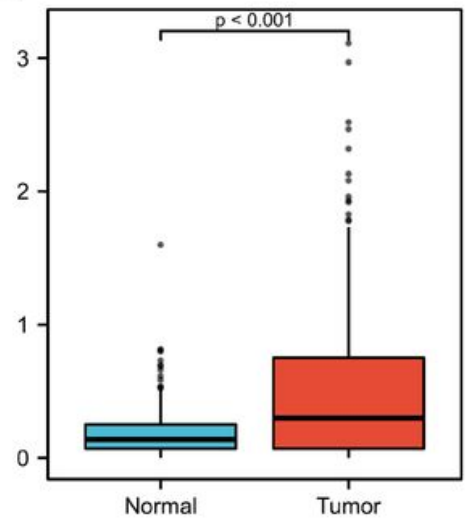

B

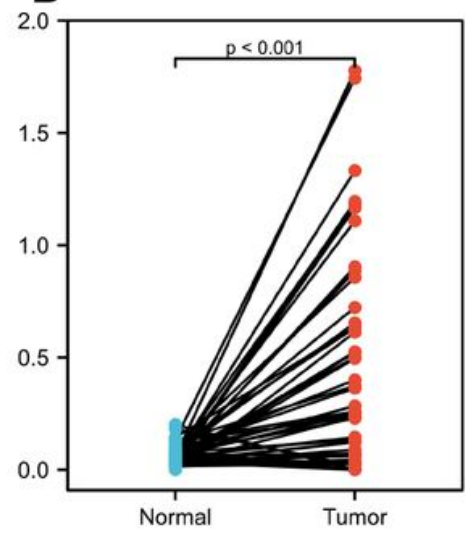

D

TSPEAR

$\begin{array}{llllll}\text { NCM-460 } & \text { SW-480 } & \text { SW-620 } & \text { HCT-116 Ht-29 LoVo }\end{array}$
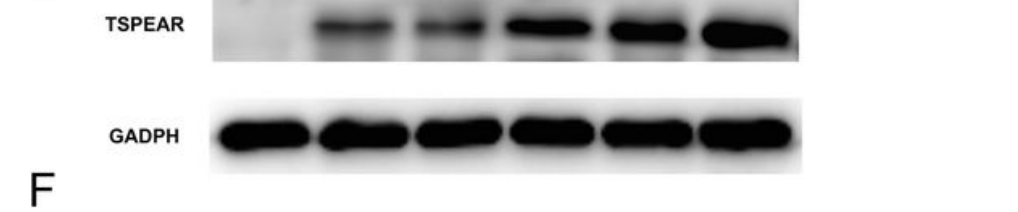

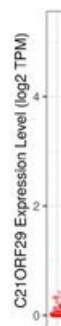

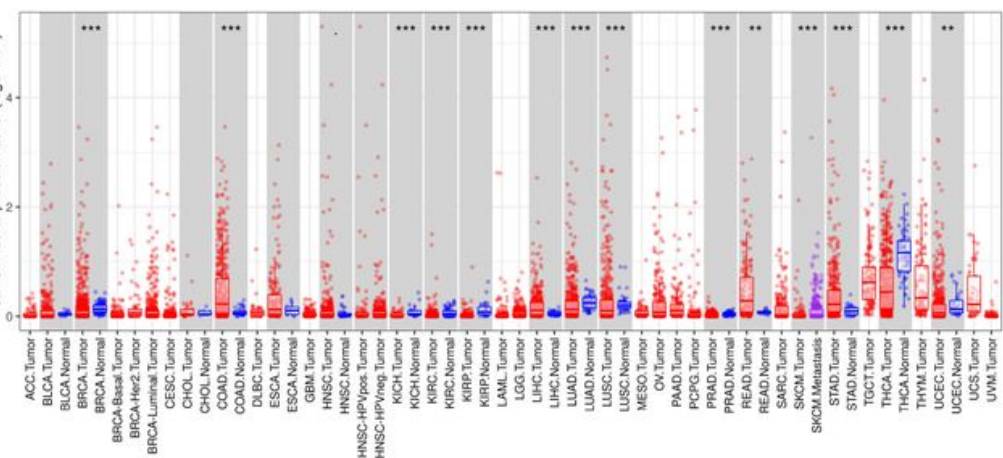

C

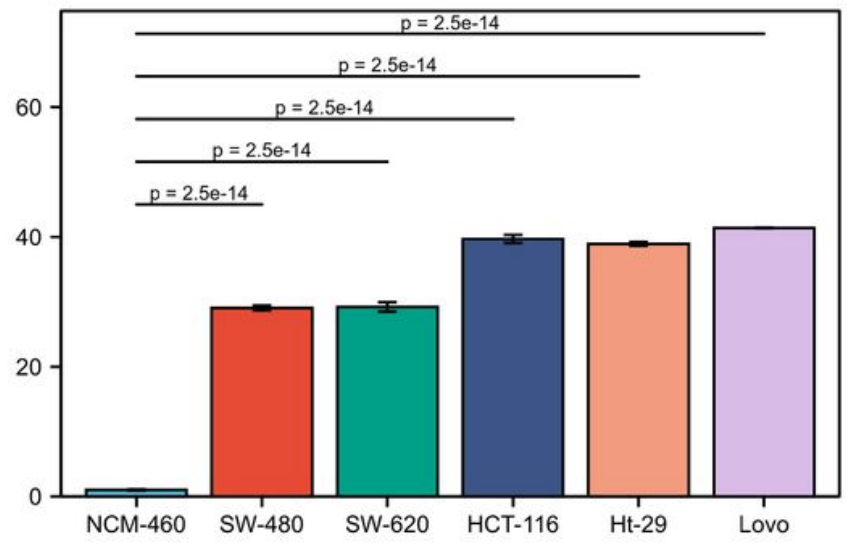

$E$

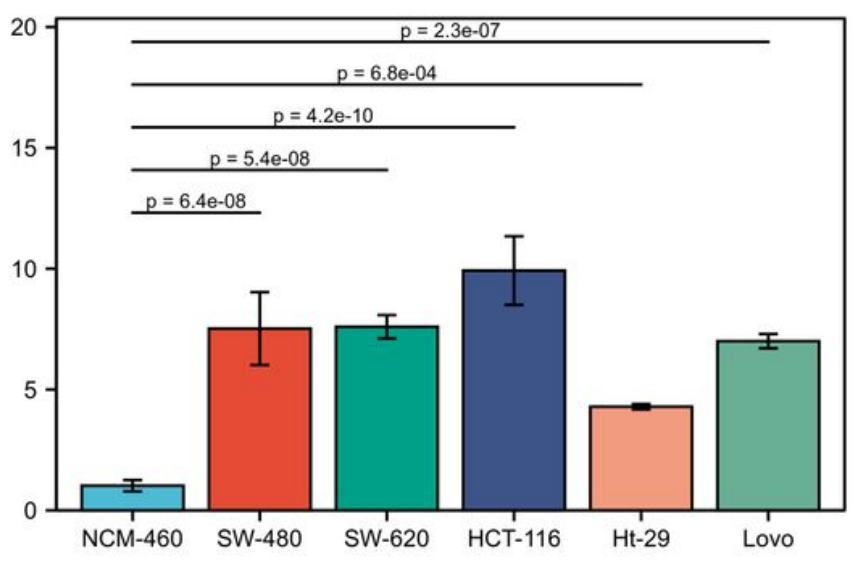

\section{Figure 1}

TSPEAR gene expression level in CRC tissues and cell lines. (A) TSPEAR expression in normal and tumor tissues. (B) TSPEAR expression in paired tissues. (C) The results of TSPEAR mRNA expression in normal cells NCM-460 and human CRC cell lines evaluated by Rt-qPCR. (D) The results of TSPEAR protein expression level in normal cells NCM-460 and human CRC cell lines by WB. (E) The result of WB in a histogram. (F)TSPEAR mRNA levels analyzed by TIMER $\left({ }^{*} p<0.05,{ }^{*} p<0.01,{ }^{* \star} p<0.001\right)$. 
A

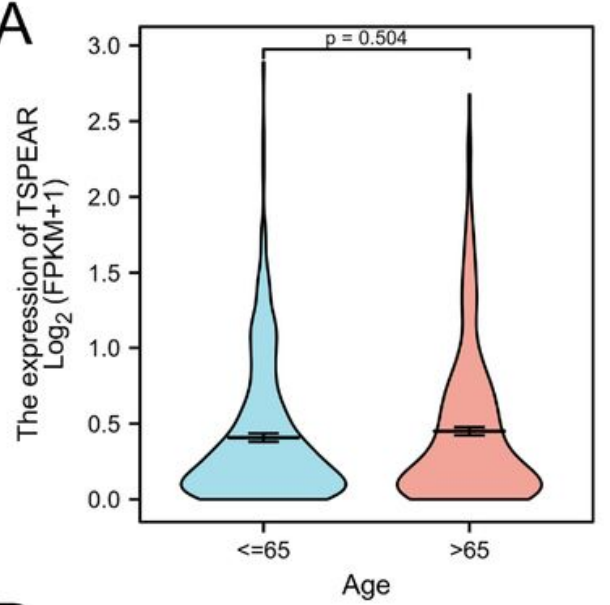

D
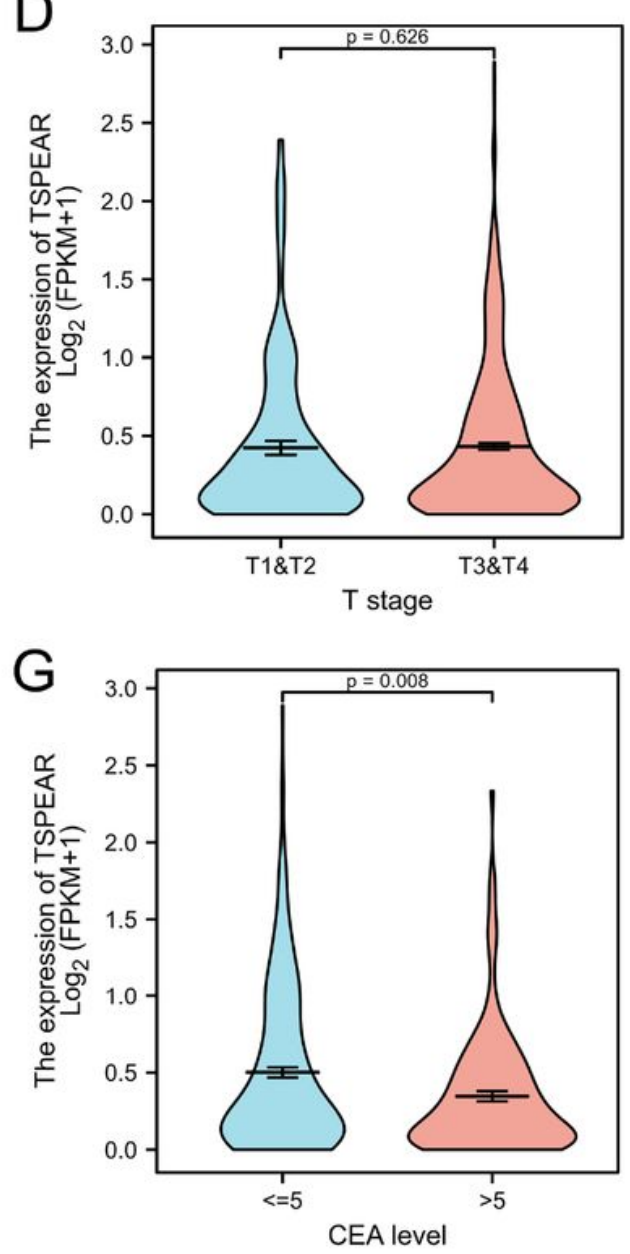

B
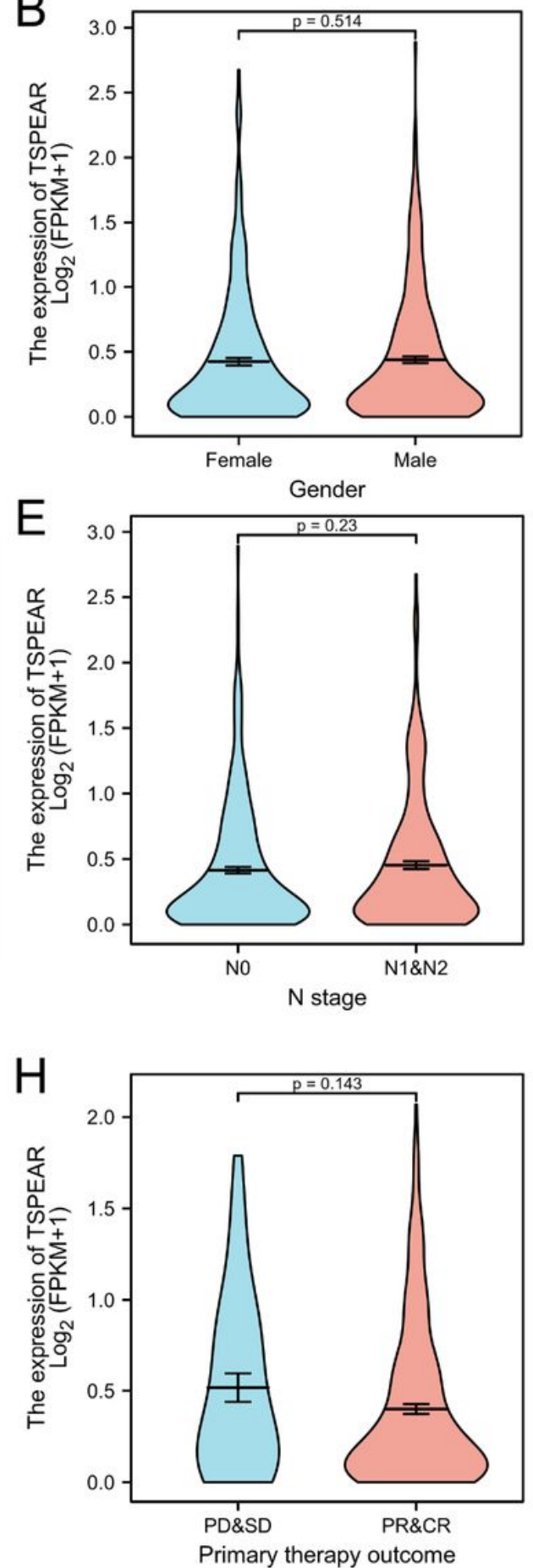

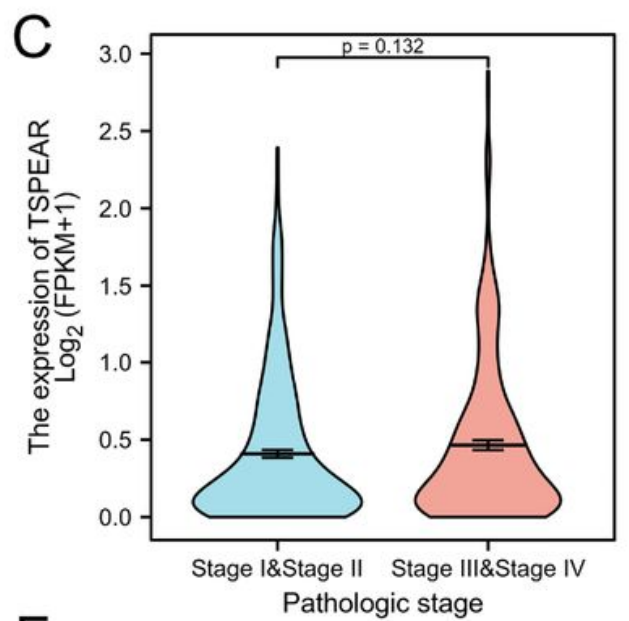

$\mathrm{F}$
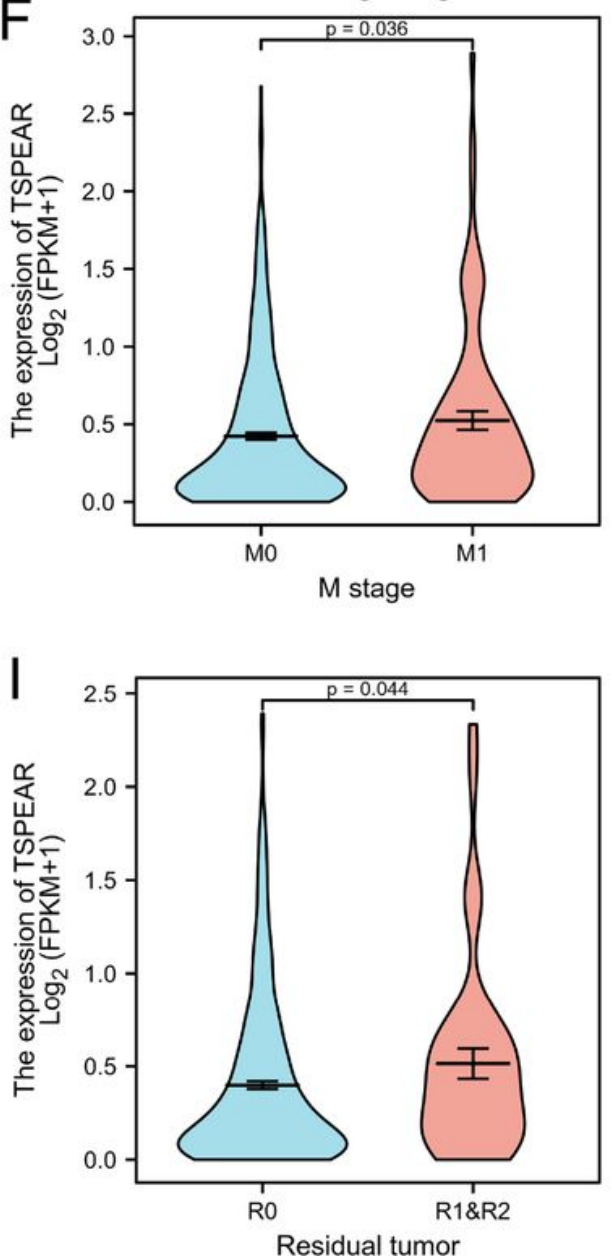

Figure 2

Association with TSPEAR expression and clinicopathological characteristics. (A) Age, (B) Gender, (C) Pathologic stage, (D) T stage, (E) N stage, (F) M stage, (G) CEA level, (H) primary therapy outcome, (I) Residual tumor. 

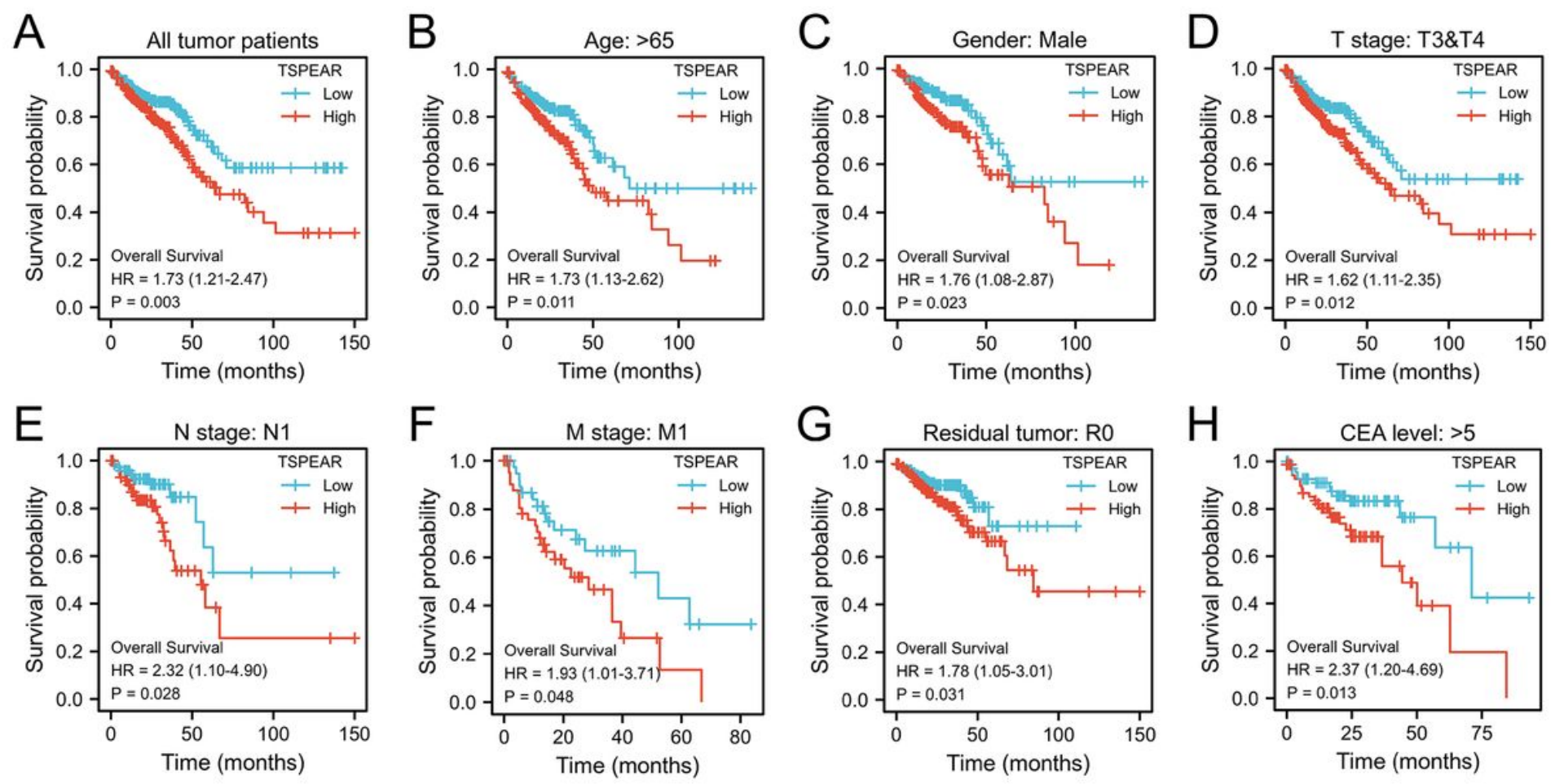

Figure 3

Kaplan-Meier curve for overall survival in colorectal adenocarcinoma. (A) Kaplan-Meier curve for TSPEAR in all tumor patients, $(B-H)$ Subgroup analysis for age greater than 65 years, Male, T3\&T4, N1, M1, R0, and CEA level more than 5 .

\begin{tabular}{|c|c|c|c|c|}
\hline Characteristics & Total(N) & $\mathrm{HR}(95 \% \mathrm{Cl})$ Multivariate analysis & & $P$ value Multivariate analysis \\
\hline Age & 619 & $2.855(1.385-5.886)$ & $E$ & 0.004 \\
\hline Pathologic stage & 619 & $7.940(1.741-36.214)$ & $\frac{1}{1}=$ & 0.007 \\
\hline T stage & 619 & $2.984(0.680-13.093)$ & $\overrightarrow{1} \longrightarrow$ & 0.147 \\
\hline $\mathrm{N}$ stage & 619 & $0.262(0.071-0.967)$ & $\frac{1}{4}$ & 0.044 \\
\hline M stage & 619 & $1.230(0.475-3.182)$ & $\begin{array}{l}1 \\
1 \\
1\end{array}$ & 0.67 \\
\hline CEA level & 619 & $1.706(0.846-3.439)$ & 4 & 0.135 \\
\hline Residual tumor & 619 & $2.114(0.925-4.831)$ & ${ }_{1}^{1}$ & 0.076 \\
\hline TSPEAR & 619 & $2.204(1.141-4.256)$ & 다 & 0.019 \\
\hline
\end{tabular}

\section{Figure 4}

Forest plot of the multivariate Cox regression analysis in colorectal adenocarcinoma. 

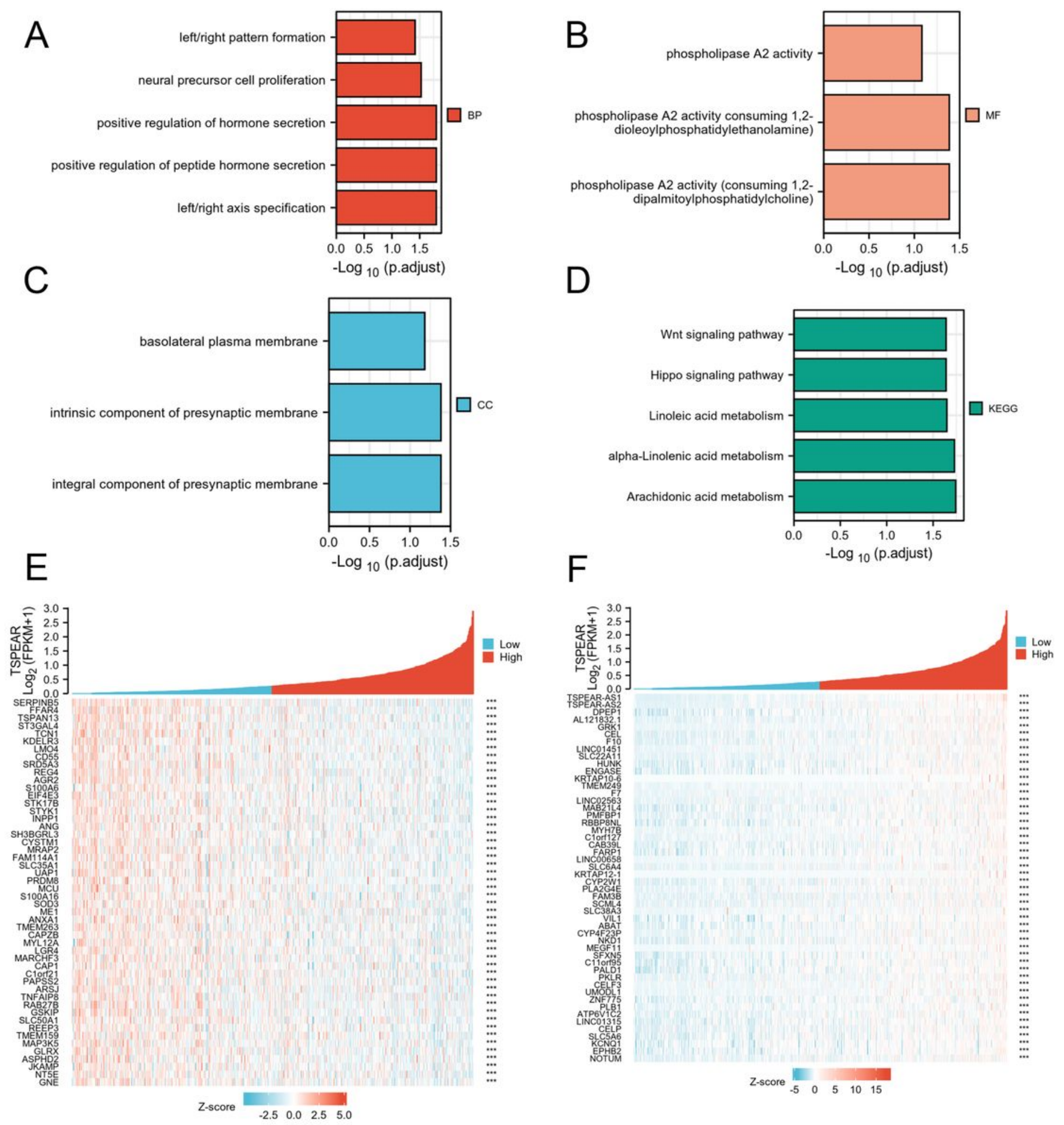

\section{Figure 5}

Gene function enrichment analysis of TSPEAR in colorectal adenocarcinoma cancer. (A-C) GO analysis, included BP, MF, CC. (D) KEGG analysis. (E) The top 50 genes most positively-correlated with TSPEAR. (F) Top 50 genes most negatively-correlated with TSPEAR. 


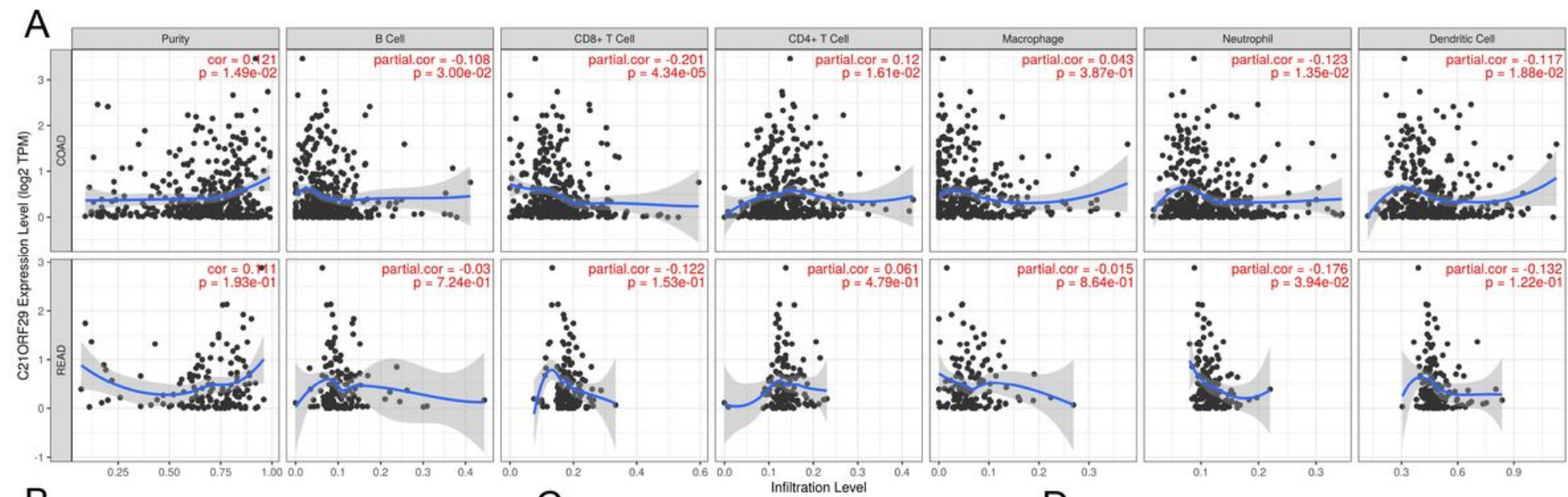

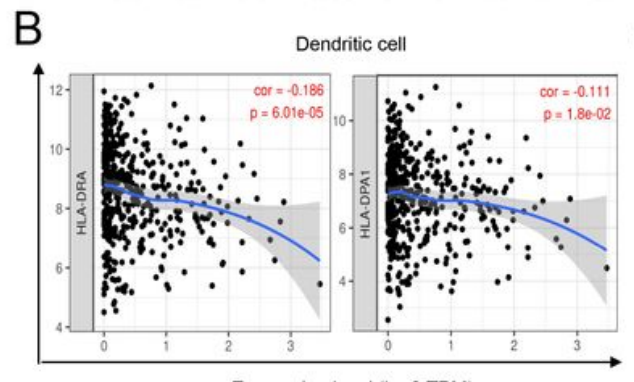

E

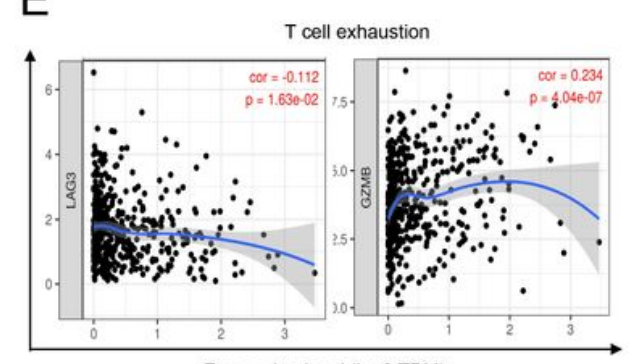

Expression level ( $\log 2$ TPM)

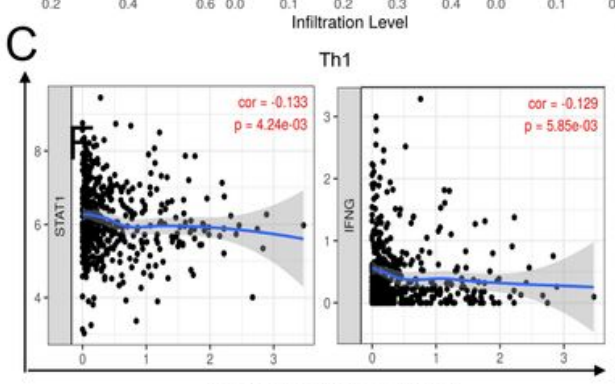

Expression level (log2 TPM)

$\mathrm{F}$

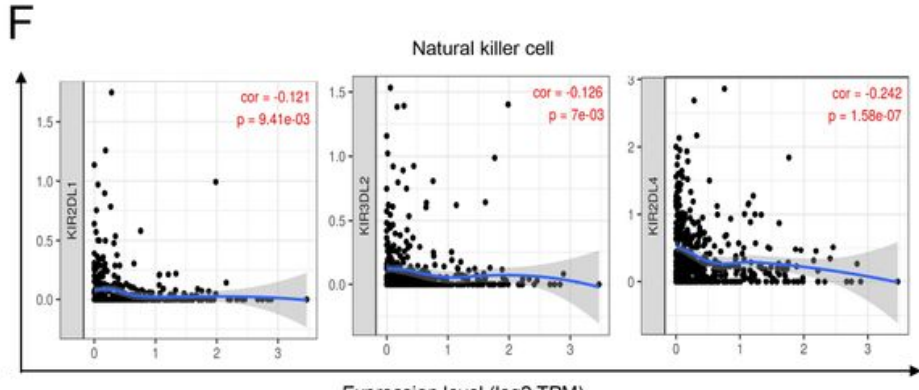

$\mathrm{D}$

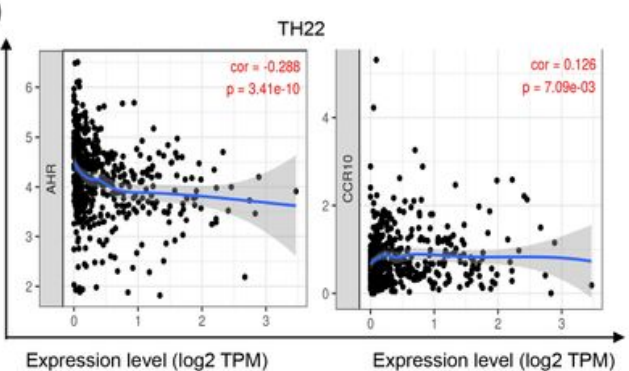

G

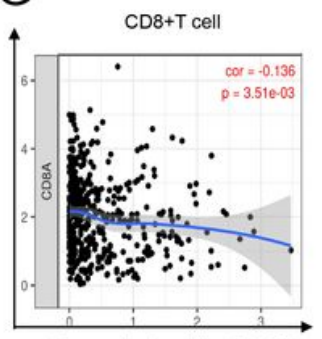

Expression level (log2 TPM)

Figure 6

Correlation of TSPEAR expression with infiltration immune in colon cancer and rectal cancer. (A). Correlations between TSPEAR expression and immune cells. (B-G). TSPEAR expression is significantly correlated with the genetic markers for Dendritic cell (HLA-DRA, HLA-DPA1), Th1 (STAT1, IFN- $\gamma$ ), TH22 (AHR, CCR10),, T cell exhaustion ( GZMB, LAG3), Natural killer cell (KIR2DL1, KIR2DL4, KIR3DL2)

and CD8+T cell (CD8A) in colon cancer.

\section{Supplementary Files}

This is a list of supplementary files associated with this preprint. Click to download.

- PCR.xIsx

- westernblotting.xIsx 